\title{
A wrench in the motor
}

\author{
Structural biology and resistance analyses reveal the binding mode and the basis for selective inhibition of the ATP \\ synthase rotary motor protein by glycosylated macrolides.
}

\section{Patrick M. M. Shelton and Tarun M. Kapoor}

M etabolic reprogramming in cancer cells imparts vulnerabilities that may be exploited by therapeutics. Certain cancer types, including leukemias and lymphomas, depend on oxidative phosphorylation (OXPHOS) in which ATP is produced by the ATP synthase rotary motor protein complex, utilizing electrochemical proton gradients in the mitochondria ${ }^{1}$. More than 250 compounds are reported to inhibit ATP synthase activity, including many natural products, and these could provide starting points for developing drugs for treating OXPHOS-dependent cancers ${ }^{2}$. However, poor therapeutic indices - the ratios of toxic to effective doses-have hindered clinical use of these compounds. In addition, a limited understanding of target engagement and binding mechanism has further impeded chemical refinement of initial leads. In this issue of Nature Chemical Biology, Reisman et al. report a combination of structural biology and deep mutational scanning-based inhibitor resistance analyses to firmly establish the physiological target and define the binding mode of glycosylated macrolides to the ATP synthase complex (Fig. 1) ${ }^{3}$. Importantly, their findings suggest how apoptolidin glycomacrolides can be further developed as therapeutics.

Modified cellular metabolism in cancer cells is well documented and is often linked to the Warburg effect, characterized by increased glucose uptake and lactose production. Despite significant effort, success in targeting cancer cell glycolysis pathways has been limited. We now recognize the heterogeneity in cancer cell energy production mechanisms, and it is becoming clear that some cancer subtypes rely more than others on upregulated mitochondrial OXPHOS pathways. Therefore, there is an increased interest in the ATP synthase complex for biochemical and cell biological studies and as a target for therapeutic discovery.

Macrolides have been studied as ATP synthase inhibitors for several decades and have been shown to be selectively cytotoxic against transformed cells. Oligomycin, for

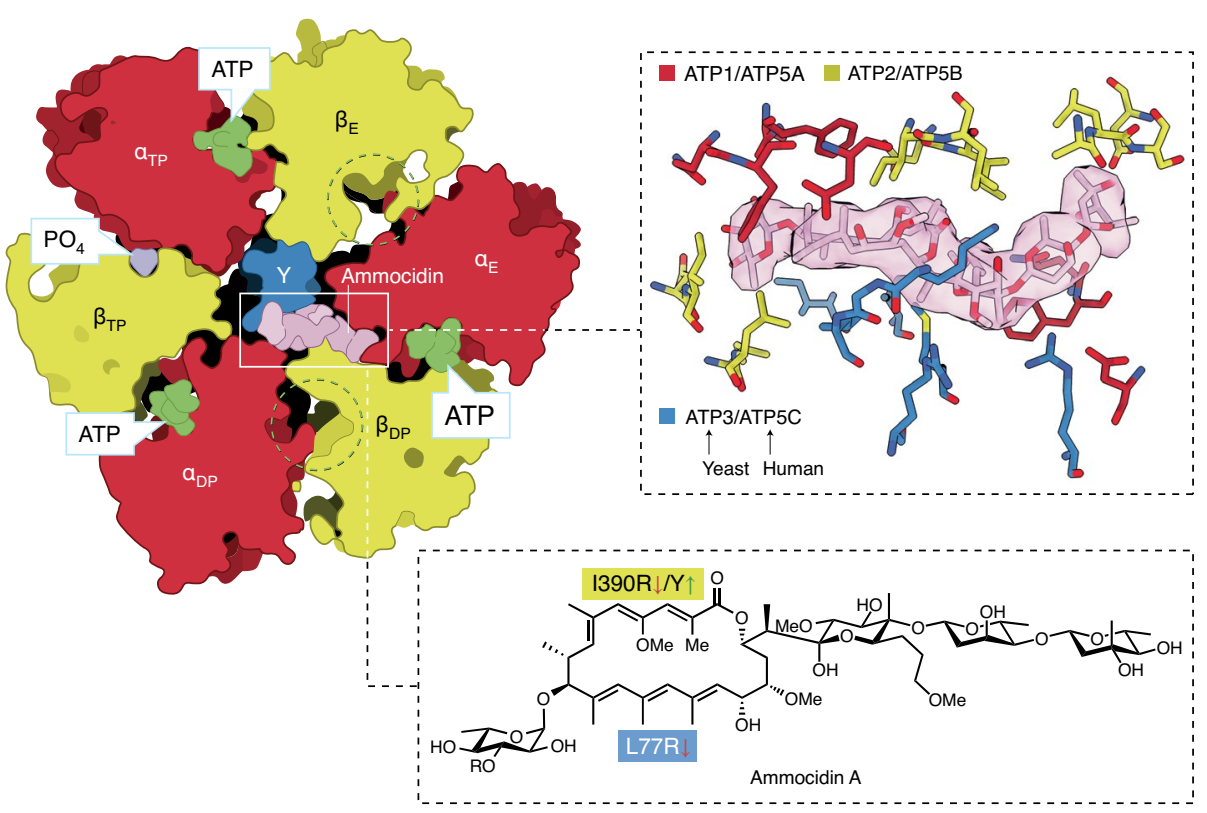

Fig. 1 | Targeting of the ATP synthase by glycomacrolides and identification of critical inhibitor interacting residues. The apoptolidins are a class of glycosylated macrolides including apoptolidin A and its close analog ammocidin A (whose chemical structure is depicted at bottom right) that were found to inhibit ATP synthase. The structure on the left is a cross-section of the cryo-EM structure of the ATP synthase-ammocidin A complex, with the ammocidin A binding site highlighted in the rectangular box. The close-up at the top right shows amino acids that define the ammocidin A binding site in ATP synthase that, when mutated, confer altered susceptibility to glycomacrolides. In particular, L77R and I390R mutations suppress glycomacrolide activity (depicted with downward red arrows near the ammocidin A structure), whereas the I390Y mutation increases sensitivity (depicted with the upward green arrow). ATP1/ATP5A, ATP2/ATP5B, and ATP3/ATP5C denote ATP synthase subunits (listed as yeast/human subunits).

example, potently inhibits ATP synthase, but toxicity and poor bioavailability have restricted its further development as a drug. The apoptolidins are a class of glycosylated macrolides with more desirable pharmacological properties. Similar to oligomycin, apoptolidin A and its close analog ammocidin A inhibit ATP synthase with nanomolar potency, although differences in their activities suggest that they have different mechanisms of action ${ }^{5}$.

To determine direct binding targets of apoptolidins, the authors synthesized photoaffinity-tagged apoptolidin analogs guided by available structure-activity relationship data ${ }^{6}$. Quantitative proteomics revealed ATP5B,a $\beta$-subunit in the catalytic $\mathrm{F}_{1}$ ring of the ATP synthase complex, to be a direct binder. Apoptolidins were uncompetitive with other $\mathrm{F}_{1}$ binding compounds, and mixed inhibition kinetics were suggestive of an allosteric binding site, which together suggest a binding mechanism distinct from that of other known ATP synthase inhibitors. Cryo-EM analyses of the apoptolidin A-ATP synthase and ammocidin A-ATP synthase complexes revealed a deep hydrophobic cavity formed by the $\alpha, \beta$ and $\gamma \mathrm{F}_{1}$ subunits in which glycomacrolides binds (Fig. 1). Reisman 
and coworkers noted a large conformational change in one of the $\beta$-subunits in the inhibitor-bound state that prevents nucleotide binding and propose that contacts across three of the seven $\mathrm{F}_{1}$ subunits directly impede the rotary mechanism of the ATP synthase complex.

Characterizing resistance can be a powerful approach to analyze compoundtarget binding in cellular contexts. When the same functionally silent mutation is shown to confer resistance to a compound in both biochemical and cell-based assays, the 'gold standard' for identification of the direct physiological target of a compound is achieved ${ }^{7}$. Reisman et al. complemented their structural studies with deep mutational scanning of ammocidin A binding site residues. The authors mutated the amino acids defining the compound binding site and identified mutations that alter sensitivity to glycomacrolides in cells. These analyses uncovered key hydrophobic interactions with the macrolide core, as L77R and I390R mutations suppressed glycomacrolide activity, while the I390Y mutation increased sensitivity (Fig. 1). Importantly, mutations conferring ammocidin A resistance did not significantly affect the function of ATP synthase. Not only do these analyses support the inhibition model derived from structural studies, but they also provide valuable information for structure-guided design to address potential resistance that may arise. Studies in mouse models with ammocidin A indicate high bioavailability, good tolerability and minimal toxicity. The promising therapeutic index of apoptolidins is unique among ATP synthase inhibitors and warrants further investigation.

Importantly, these well-characterized chemical inhibitors can be used as probes to decipher the basis of their selective toxicity to certain cancer cells.

This study exemplifies how deep mutational scanning can be combined with structural data to establish the mechanism of drug action. Deep mutational scanning is becoming an increasingly important technique for examining the links between protein sequence and function. Cancer cell genomes can be heterogeneous and unstable, and as a result, point mutations and indels within genes can be common in tumors. Powerful mutational scanning methods have been used to systematically analyze almost all possible single-nucleotide variants of tumor-suppressor genes (such as BRCA1) ${ }^{8}$. These data can provide help in interpreting variants identified by sequencing of clinical samples. Viruses can also rapidly evolve, and very recently, all possible sequence variants of the SARS-CoV-2 spike receptor binding domain have been examined to identify mutations that alter host receptor interactions and contribute to antibody escape $^{9,10}$. We expect that deep mutational scanning will be employed at early stages of therapeutic discovery not only to dissect the mode of action of a chemical inhibitor but also to better anticipate and then address drug resistance. In particular, the mutational analysis conducted by Reisman et al. should aid the design of apoptolidin analogs that not only have high efficacy but can also overcome resistance arising through mutations in the ATP synthase complex.

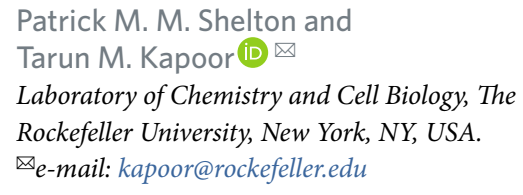

Published online: 2 December 2021

https://doi.org/10.1038/s41589-021-00897-1

References

1. Xu, Y., Xue, D., Bankhead, A. \& Neamati, N. J. Med. Chem. 63, 14276-14307 (2020).

2. Patel, B. A., D’Amico, T. L. \& Blagg, B. S. J. Eur. J. Med. Chem. 207, 112779 (2020).

3. Reisman, B. J. et al. Nat. Chem. Biol. https://doi.org/10.1038/ s41589-021-00900-9 (2021).

4. Symersky, J., Osowski, D., Walters, D. E. \& Mueller, D. M. Proc. Natl. Acad. Sci. USA 109, 13961-13965 (2012).

5. Serrill, J. D. et al. Biochem. Pharmacol. 93, 251-265 (2015).

6. Wender, P. A. et al. Org. Lett. 8, 589-592 (2006).

7. Kapoor, T. M. \& Miller, R. M. Trends Pharmacol. Sci. 38, $1100-1109$ (2017)

8. Findlay, G. M. et al. Nature 562, 217-222 (2018).

9. Star, T. N. et al. Science 371, 850-854 (2021).

10. Greaney, A. J. et al. Nat. Commun. 12, 1-14 (2021).

Competing interests

The authors declare no competing interest.

\title{
Logic invades cell-free biosensing
}

\author{
Cell-free biosensing is emerging as an effective and low-cost technology, but interpretation and synthesis of the \\ results remains largely manual. Now, researchers have incorporated a new information-processing layer between \\ biosensors and their outputs using logic gates to integrate complex results.
}

\section{Evan Amalfitano and Keith Pardee}

\begin{abstract}
L ogic gates are the fundamental core of computation, the building blocks that enable processing in all computers. These individually simple operations can be chained together to produce complex circuits capable of a huge range of processes. As the field of synthetic biology continues to advance, there has been increasing interest in using DNA-based logic gates ${ }^{1}$ in cell-free systems to merge their computational capacity with biosensing ${ }^{2}$. Now, researchers have combined the computational power of logic gates, in a new modular manner, with
\end{abstract}

small-molecule gene circuit biosensors to provide programmable logic functions and a novel take on analog-to-digital conversion ${ }^{3}$.

Cell-free biosensing is receiving increasing attention because of its ability to detect a wide variety of targets in a low-cost, portable format. In the realm of small-molecule sensing (of, for example, heavy metals or antibiotics), the recent ROSALIND (RNA output sensors activated by ligand induction) system interfaces allosteric protein transcription factors with fluorescent RNA aptamer outputs for rapid detection of over a dozen analytes in a format that can be freeze-dried for deployment ${ }^{4}$. Although this and other new systems ${ }^{5}$ are exciting, the capacity for multiplexing or logic functions has been limited. However, if, instead of an optical aptamer, protein-based sensor activation could be directed toward toehold-mediated strand displacement (TMSD) and the programmability of nucleic acid base-pairing, broad computational functionality might be realized. TMSD relies on engineered pairing of double-stranded 